\title{
Flow field of continuous phase in a vane-type pipe oil-water separator
}

\author{
Shi-ying Shi ${ }^{\mathrm{a}, \mathrm{b}}$, Jing-yu $\mathrm{Xu}^{\mathrm{a}, *}$ \\ ${ }^{a}$ LMFS, Institute of Mechanics, Chinese Academy of Sciences, Beijing 100190, China \\ ${ }^{\mathrm{b}}$ Institute of Chemistry, Chinese Academy of Sciences, Beijing 100190, China
}

\section{A R T I C L E I N F O}

\section{Article history:}

Received 24 April 2014

Received in revised form 15 September

2014

Accepted 18 September 2014

Available online 26 September 2014

\section{Keywords:}

Flow field

Pipe separator

Particle Image Velocimetry

\begin{abstract}
A B S T R A C T
In this work, the measurements of the swirling field of continuous phase in a new vane-type pipe separator have been carried out to validate the separation characteristics by the Particle Image Velocimetry. The radial, tangential and axial velocities were obtained. The results show that the new separator can successfully form a symmetrical swirling field. The transition region of the tangential velocity between the free vortex and the forced vortex shows a wider range than that in the traditional hydrocyclone. The axial velocity is always positive from the pipe wall to the central area and appears three-peak distribution. The tangential and axial velocities are dependent to the inlet flow rate, and yet show a low sensitivity to the ratio of flow split when the ratio of flow split is less than $13.1 \%$. These results are helpful to the optimal design of the downhole oil/water separator.
\end{abstract}

(c) 2014 Elsevier Inc. All rights reserved.

\section{Introduction}

With the large-scale development of deep-sea petroleum resources, the water content of output fluids is ever-increasing so that most of water treatment systems on platforms have reached its maximum capacity [1]. To solve this problem, the center for frontier engineering research (C-FER) proposed the technology of downhole oil/water separation (DOWS) which could separate the output fluids and re-injected water into the producing well [2]. This new technique has been proved to be feasible in the wells with a very high water cut. However, for the wells with high flow rate and low water cut, the processing result of the new technology is not satisfactory [3]. The reason may be due to the fact that the tangential inlet of DOWS restricts the size of hydrocyclones used in downhole. When flow rate increasing and water cut decreasing, the shear stress of fluid exerting on the oil droplet increases quickly so that the oil droplet is broken into smaller ones. To solve these problems, the technology, which the guide vane is installed in the straight pipe to generate the swirling field, attracts the attention of researchers. Gupta et al. [4] found that the straight vane could generate the swirling flow with the low level of turbulence. The following studies [5-7] showed that the guide vane with same radius in a central body could establish a symmetric flow field, and thus the droplets could be removed from gas [8-10]. In addition, Dirkzwager [11] first introduced this kind of swirl element into an oil-water separator, and successfully carried out

\footnotetext{
* Corresponding author. Tel.: +8610 8254 4179; fax: +86 1062561284

E-mail address: xujingyu@imech.ac.cn (J.-y. Xu).
}

the separation of oil and water base on the strong swirling field generated by the guide vane. However, most of studies only present qualitative information and quantitative research needs to be further investigated in detail.

In our previous work [12], a new vane-type pipe separator (VTTS) was designed as the downhole oil-water separation. Fig. 1 shows the schematic diagram of the device. The original intention of this design is to force the continuous phase to form the swirling field by the axial guide vanes installed in VTPS, which can cause the lighter dispersed phase to move toward the central area of the pipe. In this case, the water phase (continuous phase) is separated through the tangentially holes while oil phase (dispersed phase) continues to move upward. The main difference between this new separator and the traditional hydrocyclone is the inlet structure. It is well-known that the traditional hydrocyclone forms the swirling flow by a tangential slot on the side, which the VTPS is created by the axial guide vanes installed. In the present study, the swirling field of continuous phase in the VTPS was measured by the Particle Image Velocimetry (PIV), and the quantitative results were presented.

\section{Experimental}

\subsection{Flow-loop}

A VTPS with 72-mm inside diameter was constructed of plexiglass for the PIV measurements. The guide vane is composed of three semicircular plates. Each plate has a fixed angle $30^{\circ}$ with the cross section of the pipe, and occupies a half of the tangential 


\author{
Nomenclature \\ $Q_{w} \quad$ flow rate at the water-rich outlet \\ $F \quad$ ratio of flow split \\ $t$ time \\ $v_{r} \quad$ radial velocity \\ $L \quad$ vertical height from the guide vane
}

$\begin{array}{ll}\Delta t & \text { time interval } \\ Q_{i} & \text { flow rate at the inlet } \\ v_{\theta} & \text { tangential velocity } \\ v_{z} & \text { axial velocity }\end{array}$

sectional area. The thickness of the plate is $2 \mathrm{~mm}$. A schematic diagram for the installation is shown in Fig. 1. The tangential holes are distributed evenly at the cross section in the conical pipe with the same tangential velocity direction of the fluids flow. In this experiment, if the velocity vector was measured directly through the circular pipe wall, the errors from the light distortion could be caused by the pipe wall. Thus, the test section was wrapped with water in a rectangular box to reduce the errors of the distortion. This approach can make the measurement errors less than $1.6 \%$, and the same method is also adopted by other researchers to measure the swirling flow $[13,14]$.

The schematic diagram of the flow loop is illustrated in Fig. 2. Deionized water was used as the fluid, which contained silvercoated glass particles with the density of $10^{3} \mathrm{~kg} / \mathrm{m}^{3}$ and the mean diameter of $10 \mu \mathrm{m}$. Prior to the experiments, these silver-coated glass particles were evenly scattered in the water tank by stirring. And then, the water phase was fed into the VTPS under the different flow conditions. A butterfly valve was installed in the waterrich pipe to control the flow rate of the water-rich outlet. Based on an assumption that the impact of dispersed phase on the flow field of continuous phase could be ignored, the volumetric ratio of oil to water was controlled below 0.1 in this work.

The flow split of the VTPS is defined as the ratio of the flow rate of the water-rich outlet to that of the inlet:

$F=\frac{Q_{w}}{Q_{i}}$

here $F$ is the ratio of flow split, $Q_{w}$ and $Q_{i}$ are the flow rates of the water-rich outlet and the inlet, respectively.

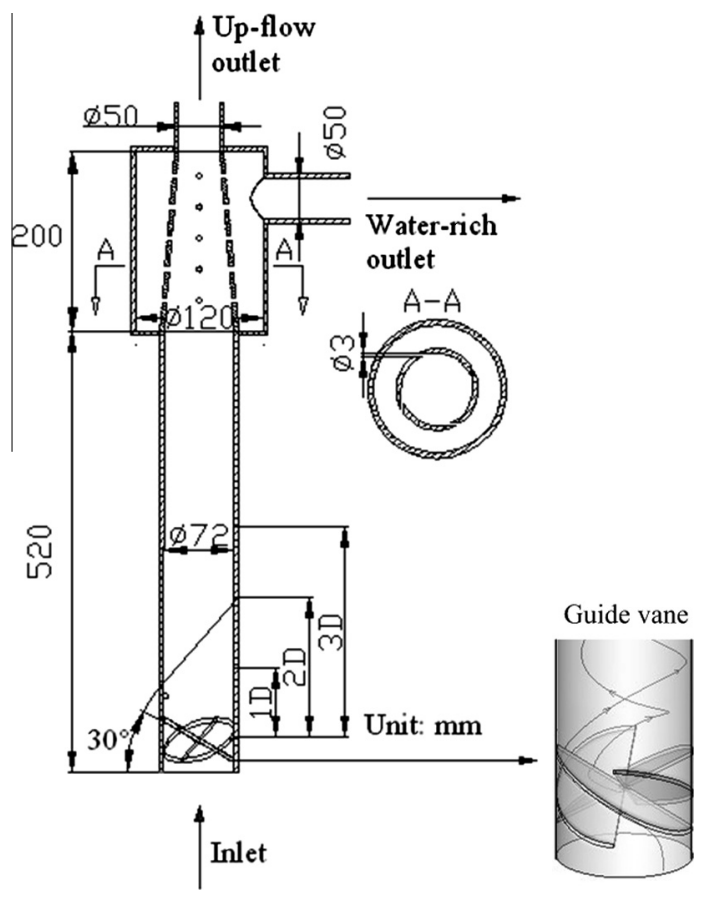

Fig. 1. The schematic diagrams of the VTPS and guide vane.

\subsection{PIV system and signal processing}

PIV system can obtain the velocity vector of the entire planes and is proved to be an effective test method. Lim et al. [15] adopted 2D PIV to study the velocity vector distribution in the hydrocyclone. Martins et al. [16] compared the typical behaviors of the tangential and axial mean velocity components got by LDA (Laser Doppler Velocimetry) with those by 2D PIV. The results showed a good agreement between two methods.

In this work, the PIV measurements were conducted with a Stereoscopic LaVision system. Short duration (4 ns) high energy $(800 \mathrm{~mJ})$ pulses of green light $(532 \mathrm{~nm})$ were ejected by a double pulsed Nd: YAG laser. The collimated laser beam transmitted through two cylindrical lens $(10 \mathrm{~mm}$ and $20 \mathrm{~mm}$ ) to generate a $1 \mathrm{~mm}$ thick light sheet. The reflected light of the reference target in the flow field was caught by a CCD camera with $1376 \times 1040$ pixels and 64-bit resolution. The cameras were furnished with Nikon $35 \mathrm{~mm} / 50 \mathrm{~mm}$ and the numerical diameter $1.8 \mathrm{~mm}$. For all the measurements, the velocity vectors were obtained by Davis 7.2 Software which processed the adaptive correlation on $64 \times 64$ pixel-size final interrogation spots with a $64 \times 64$ vectors grid. The pixel resolution was $6.45 \times 6.45 \mu \mathrm{m}$. Through the adaptive correlations, the location $r(t), \theta(t), z(t)$ of the same tracer particle were functions of time $t$. Thus, the velocity of water point that the tracer particle locates can be expressed as follows:

$v_{r}=\frac{d r(t)}{d t} \approx \frac{r(t+\Delta t)-r(t)}{\Delta t}=\overline{v_{r}}$

$v_{\theta}=\frac{d \theta(t)}{d t} \approx \frac{\theta(t+\Delta t)-\theta(t)}{\Delta t}=\overline{v_{\theta}}$

$v_{z}=\frac{d z(t)}{d t} \approx \frac{z(t+\Delta t)-z(t)}{\Delta t}=\overline{v_{z}}$

here $v_{r}, v_{\theta}, v_{z}$ are the instantaneous velocity along the radial direction, tangential direction and axial direction, respectively. $\overline{v_{r}}, \overline{v_{\theta}}, \overline{v_{z}}$ are the mean velocity. $\Delta t$ is the time interval between two continuous shooting. After the flow becomes stable, 200 samples within

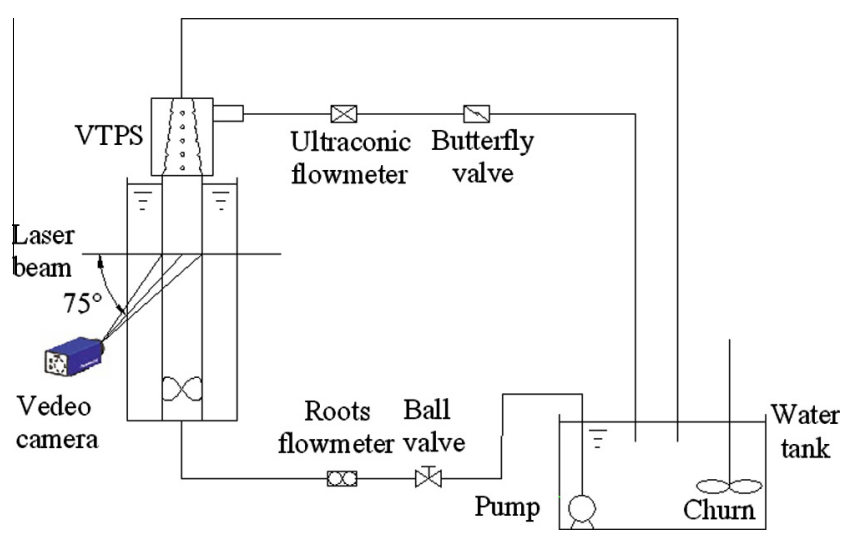

Fig. 2. Diagram of flow-loop. 
3 min are collected to obtain the time-averaged velocity at each measurement condition. Fig. 3 shows the particle images and velocity vector distribution.

In the experiments, since the VTPS was linked to the flow loop, it is difficult that the laser beam passes through the perpendicular to the video camera. Thus, there exists an angle between the video camera and the laser beam ( $75^{\circ}$ in this work). The algorithm used to reconstruct the velocity vector is tackled by the Davis 7.2 Software, and the measurement errors related to this procedure is 0.12408 pixel.

\section{Results and discussions}

As we known, the flow velocity in the hydrocyclone is composed of radial, tangential and axial velocities [17]. In the following study, three velocities will be discussed respectively.

\subsection{Radial velocity}

When the inlet flow rate is $3.0 \mathrm{~m}^{3} / \mathrm{h}$ and the ratio of flow split is 0 , Fig. 4 indicates the radial velocity distribution at different vertical heights from the guide vane. In general, the absolute value of radial velocity increases along with the radii and reaches to a maximum and then decreases rapidly to zero at the wall. The radial
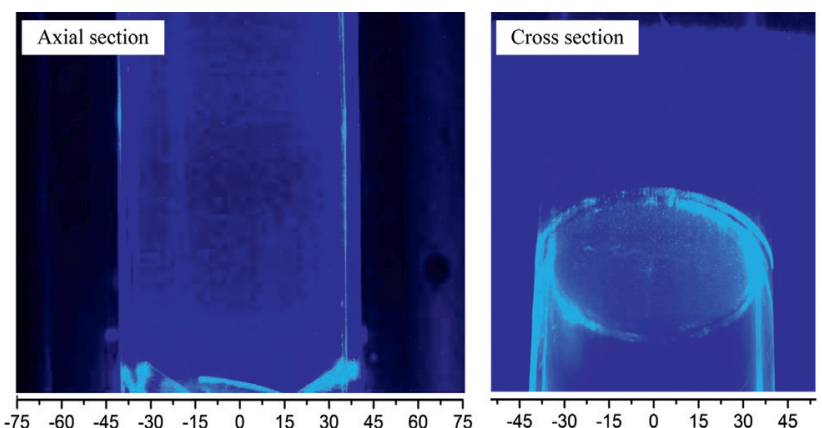

Radial position, $\mathrm{mm}$

(a) Particle images captured by the CCD

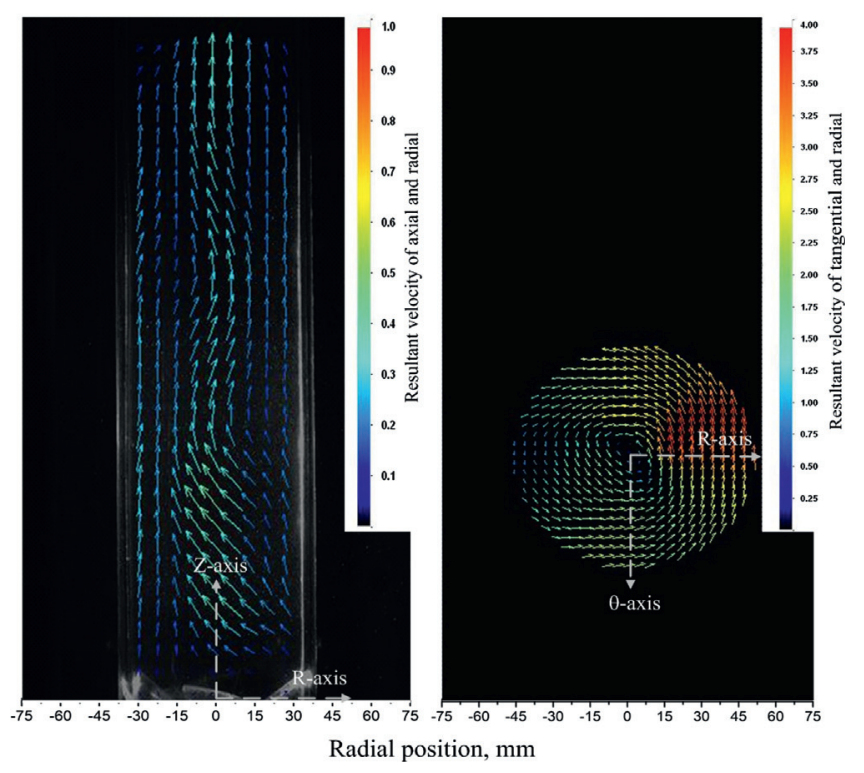

(b) Velocity vector distribution (Left: Resultant velocity of axial and radial at $Q_{i}=6 \mathrm{~m}^{3} / \mathrm{h}$, Right: Resultant velocity of tangential and radial at $Q_{i}=12 \mathrm{~m}^{3} / \mathrm{h}$ )

Fig. 3. Particle images and velocity vector distribution.

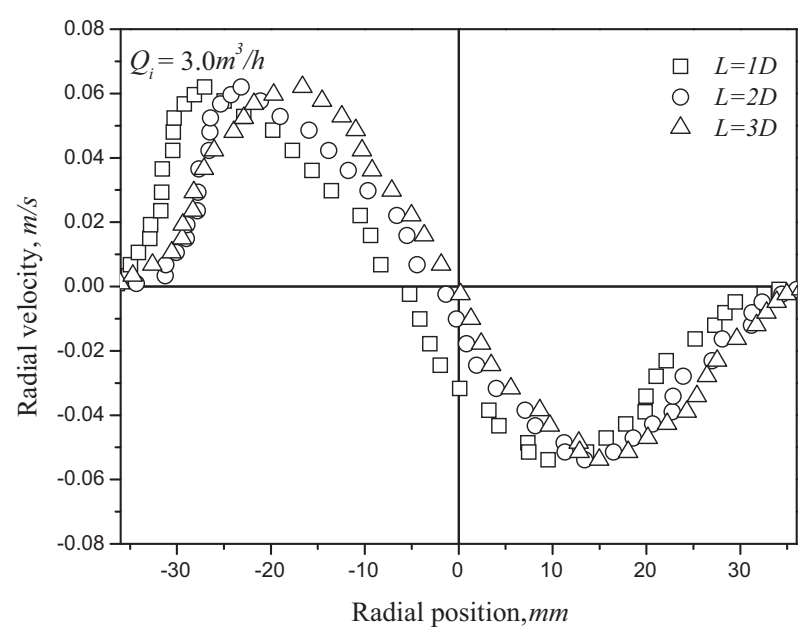

Fig. 4. Radial velocity distribution in the VTPS at different axial positions.

velocity distribution presents an approximate symmetry about the center. However, the radial velocity at the central region is far away from zero when the axial distance is less than $L=1 D$. This is due to the swirling flow after the guide vane is not a fully developed flow and not also a strictly central symmetric vector field, as is shown in the bottom of Fig. 3b. With the development distance lengthened, the flow will be stabilized, as is displayed at the top of Fig. 3b.

Compared with tangential and axial velocities, the value of radial velocity is about one order of magnitude smaller. Owing to the small values of radial velocity, measurement errors and repeatability are not satisfactory. In addition, the mean radial velocity can be obtained by a radial-axial material balance over three traverses of axial velocity whilst assuming axially symmetric flow [17]. Thus, in the following work the measurements focus on other two velocities.

\subsection{Tangential velocity}

Tangential velocity is one of the most important components inside a cyclone since the centrifugal acceleration and the centrifugal force can be obtained by its value. When the inlet flow rate is $3.00 \mathrm{~m}^{3} / \mathrm{h}$ and the ratio of flow split is 0 , Fig. 5 gives the tangential velocity distribution at the vertical height of $\mathrm{L}=2 \mathrm{D}$ from the guide

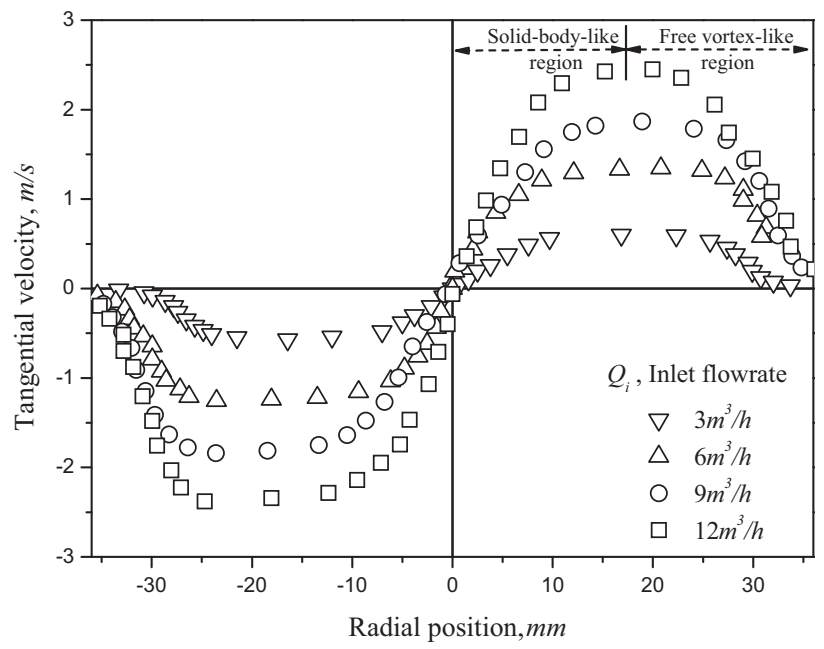

Fig. 5. Tangential velocity distribution in the VTPS at axial position $\mathrm{L}=2 \mathrm{D}$. 
vanes. It can be observed that, from the wall toward the center of the pipe, the absolute value of the tangential velocity increases quickly and then reaches to a maximum value, and finally decreases to zero. This tendency is similar to that in the radial velocity. The curve shows that the shape of a free vortex-like flows in the outer-region and solid-body-like rotation closes to the center. Note that the transition region between the free vortex and the forced vortex shows a wider range in the VTPS than that in the traditional hydrocyclone [17,18].

For an oil-water pipe separator, the inlet flow rate and the ratio of flow split are two important parameters affecting the separation efficiency. It can be found in Fig. 5 that when the ratio of flow split is zero, the tangential velocity shows a similar distribution under four different inlet flow rates. Besides, as the inlet flow rate increases, the velocity magnitude at the same radial distance increases rapidly. It indicates that the tangential velocity magnitude is dependent to the inlet flow rate. Fig. 6 shows the influence of the ratio of flow split on tangential velocity at axial position $\mathrm{L}=2 \mathrm{D}$. As is shown, an increase of the ratio of flow split causes few effect on the tangential velocity. The reason might be that the tangential velocity is measured on the cylinder section, which is located below the tapered section, and therefore the influence of the ratio of flow split on the cylinder section can be weakened. In addition, due to the limitation of the experimental conditions, the ratio of flow split is less than $13.1 \%$ so that the flow rate variation at the up-flow outlet is in a small range. However, the effects should be strengthened gradually with the ratio of flow split increasing.

\subsection{Axial velocity}

When the ratio of flow split keeps zero, the axial velocity at axial position $\mathrm{L}=2 \mathrm{D}$ is displayed in Fig. 7. As the flow rate increases, the amplitude of the axial velocity becomes large, and yet the trend of the axial velocity remains unchanged. The axial velocity is dependent to the inlet flow rate. In addition, the axial velocity is positive from the pipe wall to the central area and there is three-peak distribution in the axial velocity. The reason can be explained as follows: (1) The fluid at the central of pipe almost does not change the flow direction after the guide vane, and thus there is a peak in the central area, which is similar to the pipe flow; (2) When the radial distance increases from 0 to $36 \mathrm{~mm}$ in Fig. 5, the tangential velocity increases firstly and then reaches to the maximum. Here, the resultant velocity remains unchanged, the corresponding axial velocity will show a valley at the same radial



Fig. 6. Influence of the ratio of flow split on tangential velocity at axial position $\mathrm{L}=2 \mathrm{D}$.

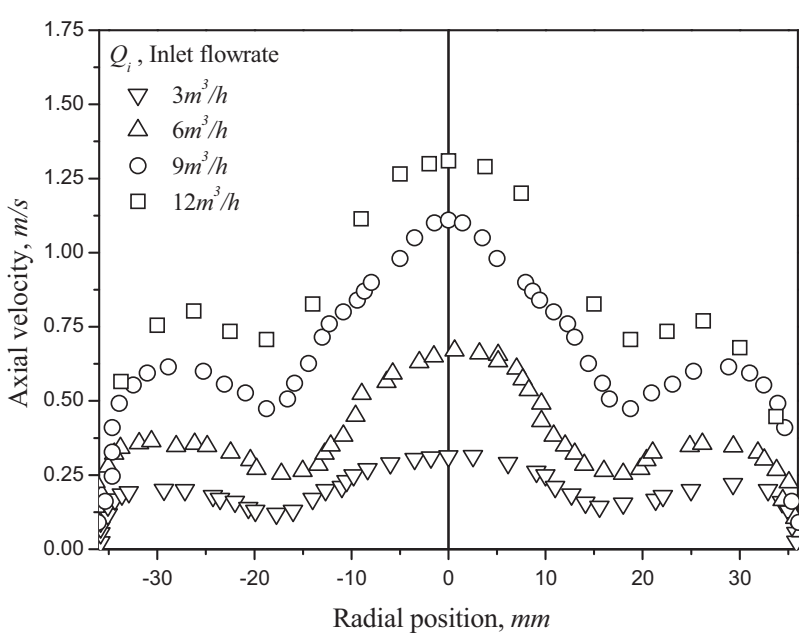

Fig. 7. Axial velocity distribution in the VTPS at axial position $\mathrm{L}=2 \mathrm{D}$.

position. And hereafter, with the tangential velocity declining, the corresponding axial velocity increases rapidly and reaches to a peak near the wall. At this time, the tangential velocity is close to zero; (3) Owing to the axis symmetry, there are two peaks for the axial velocity besides the peak in the central area. Three-peak distribution of axial velocity in the pipe swirling flow was also discovered by Swanborn [19].

Combined with the tangential velocity, it can be found that the swirling flow is formed successfully and thus the mixture of oil and water can be separated in the cross section of the pipe. Based on the different densities of oil and water, the dispersed oil phase moves toward the central area while the continuous water phase distributes close to the pipe wall. The axial velocity of the oil phase in the central area rapidly moves forward. In other words, the characteristic of axial velocity distribution helps to realize the water elimination through the tangential holes in the tapered pipe section. These are essential differences from the traditional hydrocyclone, in which the axial velocity is negative downward near the hydrocyclone wall and positive upward toward the center [20].

Based on the experimental observations, the axial velocity magnitude is hardly affected by the axial distance after the guide vanes. This is another advantage of the VTPS since the maximum value of tangential velocity is reduced significantly in the traditional hydrocylone [21]. Fig. 8 depicts the influence of the ratio of flow split on axial velocity at axial position $\mathrm{L}=2 \mathrm{D}$. When the ratio of flow split

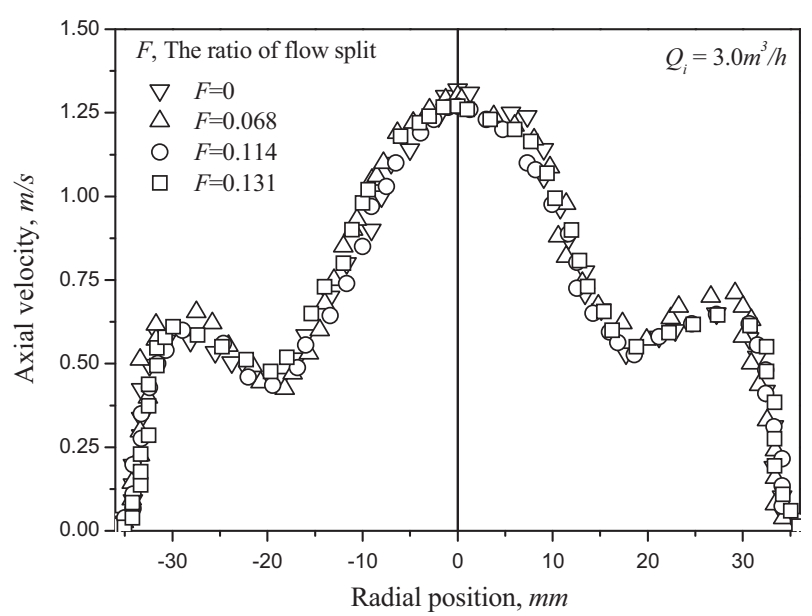

Fig. 8. Influence of the ratio of flow split on axial velocity at axial position $L=2 D$. 
increases, the axial velocity shows a few changes at the same radial distance. This means that the velocity distribution after the guide vanes is not a function of the ratio of flow split when the ratio of flow split is less than $13.1 \%$, which is the same with the tangential velocity.

\section{Conclusions}

By using the Particle Image Velocimetry System, the flow field of continuous phase was measured in a new vane-type pipe separator (VTPS). The radial, tangential and axial velocities were obtained and discussed. Through the analysis of the results, the following conclusions can be obtained:

The new guide vane can successfully form a symmetrical swirling field. Compared to the traditional flow field in the hydrocyclone, the transition region of the tangential velocity between the free vortex and the forced vortex shows a wider range in the VTPS. The axial velocity is always positive from the pipe wall to the central area and appears three-peak distribution. The tangential and axial velocities are dependent to the inlet flow rate, but show a low sensitivity for the ratio of flow split when the ratio of flow split is less than $13.1 \%$. These results are helpful to the optimal design of the DOW separator.

\section{Acknowledgments}

The authors gratefully acknowledge that the work described here is financially supported by the Special Development of National Key Scientific Instruments in China (2011YQ120048-02).

\section{References}

[1] B.E. Bowers, R.F. Brownee, P.J. Schrenkel, Development of a downhole oil/water separation and reinjection system for offshore application, SPE J. 15 (2000) $115-122$.

[2] B.R. Peachey, Downhole oil/water separation moves into high gear, J. Can. Petrol. Technol. 37 (1998) 34-41.
[3] B.R. Peachey, S. Solanki, T. Zahacy, K. Piers, Downhole oil/water separation moves into high gear, in: 48th Annual Technical Meeting of the Petroleum Society, Calgary, 1997, pp. 97-91.

[4] A.K. Gupta, D.G. Lilley, N. Syred, Swirl Flows, Gordon \& Breach Science Pub, USA, 1984.

[5] C. Verlaan, Performance of Novel Mist Eliminators, Ph.D. Thesis, Delft University of Technology, 1991.

[6] F. Bario, C. Beral, Boundary layer measurements on the pressure and suction sides of a turbine inlet guide vane, Exp. Therm. Fluid Sci. 17 (1998) 1-9.

[7] T.-M. Liou, H.-L. Lee, C.-C. Liao, Effects of inlet guide-vane number on flow fields in a side-dump combustor, Exp. Therm. Fluid Sci. 24 (2001) 11-23.

[8] F.T.M. Nieuwstadt, M. Dirkzwager, A fluid mechanics model for an axial cyclone separator, Ind. Eng. Chem. Res. 34 (1995) 3399-3404.

[9] B. Cai, J. Wang, L. Sun, N. Zhang, C. Yan, Experimental study and numerical optimization on a vane-type separator for bubble separation in TMSR, Prog. Nucl. Energy 74 (2014) 1-13.

[10] Z. Xiong, M. Lu, M. Wang, H. Gu, X. Cheng, Study on flow pattern and separation performance of air-water swirl-vane separator, Ann. Nucl. Energy 63 (2014) 138-145.

[11] M. Dirkzwager, A New Axial Cyclone Design for Fluid-Fluid Separation, Ph.D. Thesis, Delft University of Technology, USA, 1996.

[12] S.Y. Shi, J.Y. Xu, H.Q. Sun, J. Zhang, D.H. Li, Y.X. Wu, Experimental study of a vane-type pipe separator for oil-water separation, Chem. Eng. Res. Des. 90 (2012) 1652-1659.

[13] C.C. Che, M.C. Tian, G.M. Zhang, X.L. Leng, Experimental study by PIV of swirling flow induced by trapezoid-winglets, J. Hydrodynamics 25 (2013) 919-928.

[14] F.C. Li, Y. Dong, Y. Kawaguchi, M. Oshima, Experimental study on swirling flow of dilute surfactant solution with deformed free-surface, Exp. Therm. Fluid Sci. 33 (2008) 161-168.

[15] E.W.C. Lim, Y.-R. Chen, C.-H. Wang, R.-M. Wu, Experimental and computation studies of multiphase hydrodynamics in a hydrocyclone separator system, Chem. Eng. Sci. 65 (2010) 6415-6424.

[16] L.P.M. Martins, D.G. Duarte, J.B.R. Loureiro, C.A.C. Moraes, A.P. Silva Freire, LDA and PIV characterization of the flow in a hydrocyclone without an air-core, J. Petrol. Sci. Eng. 70 (2010) 168-176.

[17] J. Bergström, H. Vomhoff, Experimental hydrocyclone flow field studies, Sep. Purif. Technol. 53 (2007) 8-20.

[18] D.F. Kelsall, A study of the motion of solid particles in a hydraulic cyclone Trans. Inst. Chem. Eng. 30 (1952) 87-108.

[19] R.A. Swanborn, A New Approach to The Design of Gas-Liquid Separators for The Oil Industry. Ph.D. Thesis, Delft University of Technology, 1988.

[20] B. Dabir, C.A. Petty, Measurement of mean velocity profiles in a hydrocyclone using laser Doppler anemometry, Chem. Eng. Commun. 48 (1986) 377-388.

[21] Z.S. Bai, H.L. Wang, S.T. Tu, Experimental study of flow patterns in deoiling hydrocylones, Miner. Eng. 22 (2009) 319-323. 